\section{Subacute thyroiditis after seasonal influenza vaccination}

\author{
Fumiatsu Yakushiji, ${ }^{1}$ Kenji Ohnishi, ${ }^{2}$ \\ Mutsuko Yasuda, ${ }^{1}$ Taiichiro Kobayashi, ${ }^{1}$ \\ Hiroyuki Kinoshita ${ }^{1}$ \\ 'Department of Internal Medicine, Tokyo \\ Metropolitan Bokutoh Hospital, Tokyo; \\ 2Department of Infectious Diseases, \\ Tokyo Metropolitan Bokutoh Hospital, \\ Tokyo, Japan
}

\section{Abstract}

We report the first patient with subacute thyroiditis after influenza vaccination in Japan. A 49-year-old woman received a seasonal influenza vaccination in October 2009. She also received a seasonal influenza vaccination in 2008. After the vaccination, she experienced spontaneous pain and tenderness in the right lower neck. Together with the results of laboratory examinations and thyroid echography, we established the diagnosis of subacute thyroiditis. The patient had human leukocyte antigens (HLAs) of A2/A11 and B62/B35. HLA-A2-positive is reported at a risk of interferon-alphainduced autoimmune thyroid disorder, and HLA-B35-positive is reported at a risk of subacute thyroiditis. Since fever and cervical pain are reported as adverse effects of influenza vaccination, subacute thyroiditis after influenza vaccination might have been missed.

\section{Introduction}

Influenza vaccination has well-established effects against influenza virus infection. ${ }^{1}$ However, various complications have been reported as adverse effects of this vaccination. ${ }^{1}$ Here, we report the first patient with subacute thyroiditis after influenza vaccination in Japan, and discuss the possible involvement of the human leukocyte antigen (HLA).

\section{Case Report}

A 49-year-old woman with no history of thyroid disease received a seasonal influenza vaccination (influenza hemagglutinin (HA) vaccine; SEIKEN ${ }^{\circledR}$, Takeda Pharmaceutical Co. Ltd., Tokyo, Japan) in October 2009. She also received a seasonal influenza vaccination in 2008. The patient does not have any drug allergies, including allergies against vaccinations. At the time of vaccination, she did not have a cold. At 2 days after vaccination, she experienced spontaneous pain and tenderness in the right lower neck, however, she did not feel a fever. After 9 days, the pain worsened, and her body temperature became approximately $37.0^{\circ} \mathrm{C}$. She experienced general fatigue and shortness of breath when she walks fast. After 16 days, the pain worsened and radiated to the right ear. After 22 days, the patient visited our hospital. Physical examinations showed no anemia, no lung rale, no cardiac murmur, and no arrhythmia. The thyroid was not enlarged but exhibited tenderness. Laboratory examinations including urinalysis, cell blood count (CBC) and blood biochemical test revealed mild inflammation: white blood cell count (WBC), 7400 cells $/ \mu \mathrm{L}$ (reference, 270010,300); neutrophil, 71.7\% (reference, 37.080.0); C-reactive protein (CRP), $1.02 \mathrm{mg} / \mathrm{dL}$ (reference, $<0.30$ ); and erythrocyte sedimentation rate (ESR), $69 \mathrm{~mm} /$ hour (reference, 210). Parameters describing thyroid function and thyroid antibodies were as follows: thyroid stimulating hormone (TSH), $<0.01 \mu \mathrm{U} / \mathrm{mL}$ (reference, 0.35-4.94); free triiodothyronine (FT3), $6.33 \mathrm{pg} / \mathrm{mL}$ (reference, 1.71-3.71); free tetraiodothyronine (FT4), $2.60 \mathrm{ng} / \mathrm{dL}$ (reference, 0.70-1.48); thyroglobulin, $603.6 \mathrm{ng} / \mathrm{mL}$ (reference, <32.7). Thyroid antibodies were negative. Thyroid ultrasonography with color doppler revealed low bloodstream area throughout the thyroids, and a hypoechogenic area in the right lobe of the thyroid. This hypoechogenic area corresponded to the area of spontaneous pain, and probe touching worsened the pain (Figure 1). The patient tested positive for the following human leukocyte antigens (HLAs) by lymphocyte analysis: $\mathrm{A} 2 / \mathrm{A} 11, \mathrm{~B} 62 / \mathrm{B} 35, \mathrm{DR} 4 / \mathrm{DR} 14=\mathrm{DRB} 1 * 04$ DRB $1 * 14$.

On the basis of the history of illness, laboratory results, and the results of thyroid echography, we diagnosed the patient with subacute thyroiditis using the criteria for subacute thyroiditis of the Japan Thyroid Association. ${ }^{2}$ After the administration of predonisolone 10 $\mathrm{mg}$ /day, the patient's symptoms improved.

\section{Discussion}

According to a standard textbook of Endocrinology, subacute thyroiditis is thought to be caused directly or indirectly by various viral infections, including influenza virus; ${ }^{3}$ therefore, influenza vaccine may cause subacute thyroiditis. Since the influenza vaccine is an inactive vaccine, direct mechanisms, such as virus proliferation, may not be involved. Instead, indirect mechanisms, such as immune responses, may be involved in the subacute thyroiditis in the present patient.

As reported previously, HLA-A1, A2, B8, and
Correspondence: Hiroyuki Kinoshita, Department of Internal Medicine, Tokyo Metropolitan Bokutoh Hospital, 4-23-15 Koutohbashi, Sumida-ku, Tokyo 130-8575, Japan. Tel. +81.3.3633.6151 - Fax: +81.3.3633.6173

E-mail: hkinoshita-tky@umin.ac.jp

Key words: subacute thyroiditis, influenza, vaccination, human leukocyte antigen.

Contributions: FY, patient diagnosis and treatment; K0, TK, clinical suggestion between influenza virus and its complications; MY, patient medical care; HK, manuscript writing, patient medical care.

Conflict of interest: the author reports no conflicts of interest.

Received for publication: 15 June 2011. Revision received: 16 July 2011.

Accepted for publication: 18 July 2011.

This work is licensed under a Creative Commons Attribution NonCommercial 3.0 License (CC BYNC 3.0).

(C) Copyright F. Yakushiji et al., 2011

Licensee PAGEPress, Italy

Drugs and Therapy Studies 2011; 1:e10

doi:10.4081/dts.2011.e10

B35 enhance influenza A virus-specific cytotoxic T-lymphocyte response, and HLA-A2 has the strongest effect. ${ }^{4}$ In patients with chronic hepatitis C, HLA-A2, B46, and Cw7 were associated with an interferon-alpha-induced autoimmune thyroid disorder; especially, the relative risk of HLA-A2 was 10.2. ${ }^{5}$ Further, subacute thyroiditis was associated with HLA B-35 and B-67; especially, the relative risk of HLAB35 was 18.02. ${ }^{6}$ Since our patient showed HLAA2/A11 B62/B35, we suppose that her HLA was related to the subacute thyroiditis after

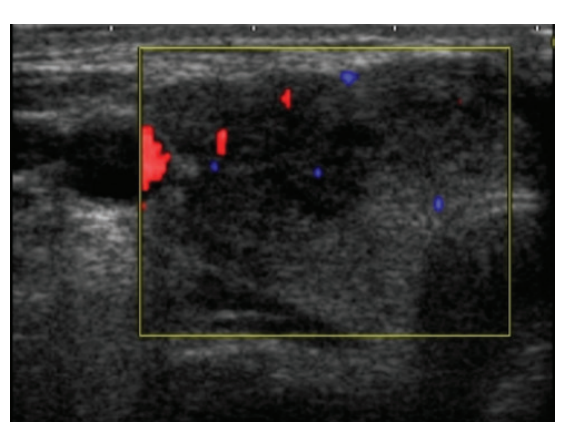

Figure 1. Thyroid ultrasonography (Color Doppler): the low bloodstream area throughout the thyroids, and a hypoechogenic area in the right lobe of the thyroid. The hypoechogenic area corresponded to the area of spontaneous pain, and probe touching worsened the pain. 
influenza vaccination. There have been two case reports of subacute thyroiditis after influenza vaccination. ${ }^{7,8}$ However, HLA was not reported in both case reports. Since fever and cervical pain are reported as adverse effects of influenza vaccination, ${ }^{9}$ subacute thyroiditis after influenza vaccination might have been missed. Medical practitioners should be aware of subacute thyroiditis when administering influenza vaccine to patients.

\section{References}

1. Fiore AE, Shay DK, Broder K, et al. Centers for Disease Control and Prevention. Prevention and control of seasonal influenza with vaccines: recommenda- tions of the Advisory Committee on Immunization Practices (ACIP), 2009. MMWR Recomm Rep 2009;58:1-52.

2. Guidelines for the Diagnosis of Subacute Thyroiditis. The Japan Thyroid Association. Available from: http://www. japanthyroid.jp/doctor/guideline/english.ht ml\#akyuu. Accessed June 15, 2011.

3. Henry K, Shlomo M, Kenneth P, L. Reed. Williams Textbook of Endocrinology, 11th edition. Philadelphia: Saunders; 2007. p.366.

4. Boon AC, de Mutsert G, Graus YM, et al. The magnitude and specificity of influenza A virus-specific cytotoxic T-lymphocyte responses in humans is related to HLA-A and -B phenotype. J Virol 2002;76:582-90.

5. Kakizaki S, Takagi H, Murakami M, et al. HLA antigens in patients with interferonalpha-induced autoimmune thyroid disor- ders in chronic hepatitis C. J Hepatol 1999;30:794-800.

6. Ohsako N, Tamai H, Sudo T, et al. Clinical characteristics of subacute thyroiditis classified according to human leukocyte antigen typing. J Clin Endocrinol Metab 1995;80:3653-6.

7. Hsiao JY, Hsin SC, Hsieh MC, et al. Subacute thyroiditis following influenza vaccine (Vaxigrip) in a young female. Kaohsiung J Med Sci 2006;22:297-300.

8. Girgis CM, Russo RR, Benson K. Subacute thyroiditis following the H1N1 vaccine. J Endocrinol Invest 2010;33:506.

9. Tremblay ME, Closon A, D'Anjou G, Bussières JF. Guillain-Barré syndrome following H1N1 immunization in a pediatric patient. Ann Pharmacother 2010;44:13303. 